\title{
Pleomorphic liposarcoma of the chest wall: A case report
}

\author{
Delandy H.Y. McConnell ${ }^{1}$, David St. Michel ${ }^{1}$, Alan J. Shienbaum² ${ }^{* 2}$ Amrit Nayar ${ }^{1}$, Darshan Roy ${ }^{2}$ \\ ${ }^{1}$ Department of General Surgery, Rowan University School of Osteopathic Medicine, Stratford, New Jersey, United States \\ ${ }^{2}$ Department of Pathology, Rowan University School of Osteopathic Medicine, Stratford, New Jersey, United States
}

Received: September 12, 2017

Accepted: November 5, 2017

Online Published: November 7, 2017

DOI: $10.5430 /$ css.v3n4p19

URL: https://doi.org/10.5430/css.v3n4p19

\begin{abstract}
Liposarcomas represent the most common type of soft tissue sarcoma. Liposarcoma is sub-classified into dedifferentiated, myxoid, pleomorphic, and unspecified types, with the pleomorphic form being the most rare. The most common locations of this tumor are the popliteal fossa, retroperitoneum, neck and genitourinary system. Primary axillary, chest wall and intrathoracic origin of this lesion is extremely rare with few studies available to guide treatment. This study describes the wide surgical excision of a pleomorphic liposarcoma of the chest wall, which was successfully resected after obtaining magnetic resonance imaging, computed tomographic angiogram, and positron emission tomography scan. We also describe how the definitive diagnosis was established by histopathologic analysis.
\end{abstract}

Key Words: Sarcoma, Pleomorphic liposarcoma, Chest wall, Liposarcoma

\section{INTRODUCTION}

Liposarcomas are malignancies that represent $20 \%-30 \%$ of all adult soft tissue tumors. ${ }^{[1]}$ Most are asymptomatic, but a small percentage present with mass effects such as pain and obstruction. ${ }^{[1]}$ Most commonly, liposarcoma arises in the retroperitoneum, lower extremities (particularly popliteal fossa), neck, and genitourinary organs. Primary axillary, chest wall or intrathoracic origin of this entity is very rare. A subclass of these tumors includes pleomorphic liposarcoma, which are extremely rare in the axillary and thoracic region. This case report aims to describe the diagnosis of this particularly rare soft tissue tumor and presents wide margin excision as treatment option.

\section{Presentation of Case}

A 61-year-old male presented from his primary care physician with a rapidly-growing right axillary mass. He first noticed the mass five to six months previously and noted a significant increase in size. Per patient report, since initial recognition, the mass had more than doubled in size and now was causing pain in the triceps region. The patient reported no recent weight loss, anorexia or other symptoms. Physical examination revealed a $12 \mathrm{~cm}$ mass in greatest dimension, which was firm, fixed and extending from the axillary apex to the mid-axillary chest wall. No skin changes, edema or tenderness were present. The patient had a previous surgery of the right axilla for hyperhidrosis $10-15$ years prior to this presentation.

\section{Results}

\subsection{Imaging findings}

Magnetic Resonance Imaging (MRI) of the chest revealed a heterogeneous right axillary mass measuring $10 \mathrm{~cm}-12 \mathrm{~cm}$ in greatest dimension, possibly invading the right axillary vein

\footnotetext{
*Correspondence: Alan J. Shienbaum; Email: shienbaj@ rowan.edu; Address: Kennedy University Hospital - Cherry Hill, Department of Pathology, 2201 Chapel Avenue West, Cherry Hill, NJ 08002, United States.
} 
with development of venous collateral circulation in the chest wall (see Figure 1). Subsequent Computed Tomographic Angiogram (CTA) of the chest revealed a heterogenous large $12 \mathrm{~cm}$ lobulated axillary/right lateral chest wall mass extending to the apex of the axilla with invasion of the right axillary vein, and possible invasion of the right latissimus dorsi and the teres major muscle (see Figure 2). A Positron Emission Tomography (PET) scan performed two months after the wide resection of the right axillary/chest wall lesion demonstrated a $12 \mathrm{~mm}$ lobulated nodule present in the left lower lobe of the lung abutting the posterior cardiac border. This lung lesion showed marked hypermetabolism measuring a maximum SUV of 6.2, which was reported as suspicious for malignancy. No evidence of recurrence of the right axillary/chest wall lesion was identified on the PET scan performed two month after the initial resection.
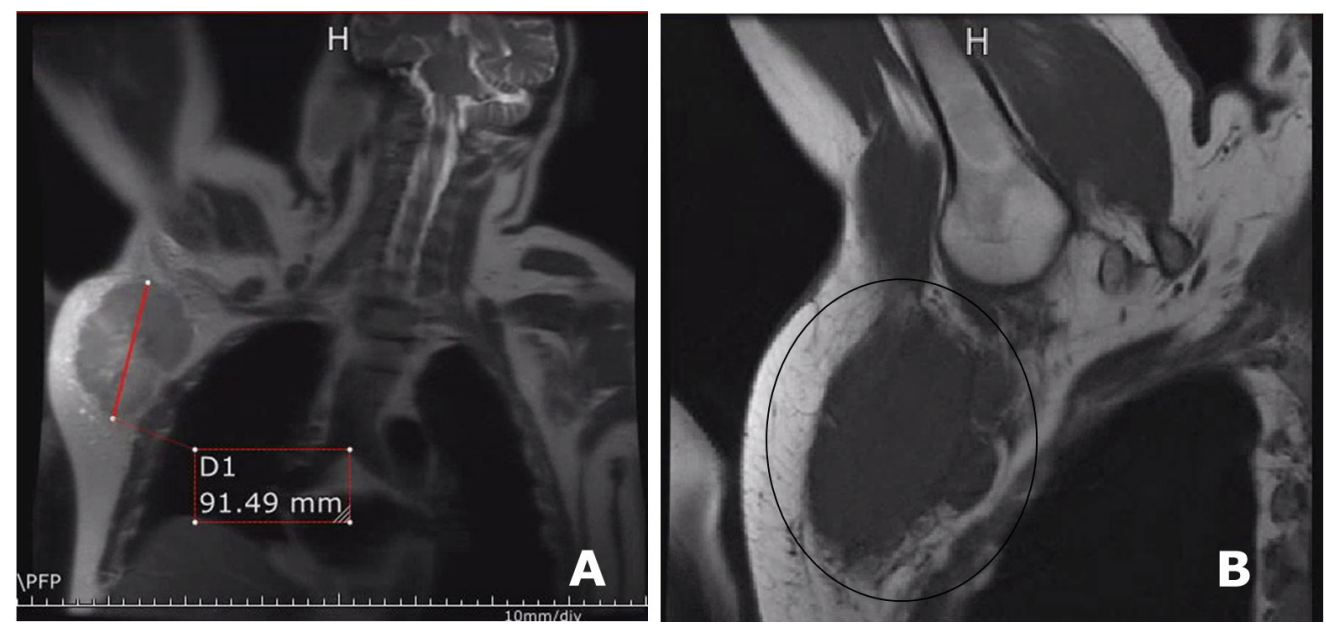

Figure 1. Magnetic resonance imaging showed a heterogeneous mass with lobulated contours measuring $10.9 \mathrm{~cm}$ in AP $\times 8.3 \mathrm{~cm}$ in transverse $\times 10 \mathrm{~cm}$ in craniocaudal diameter (A and $\mathrm{B}$ ); This mass demonstrates intermediate to low T1-weighted signal and heterogeneously increased T2 signal
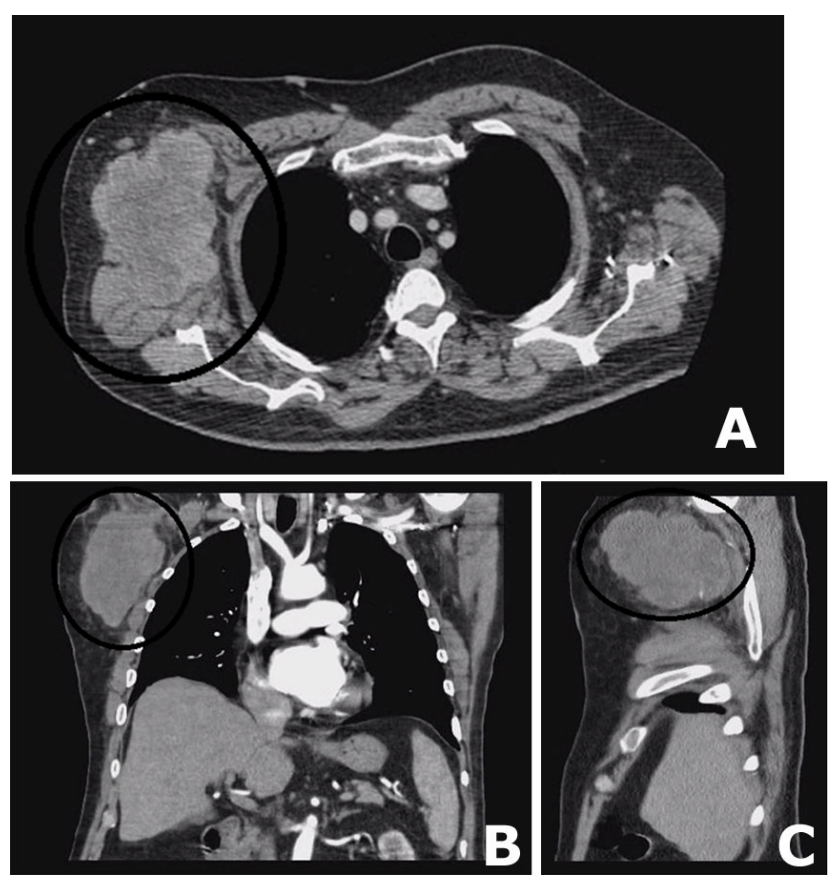

Figure 2. Computed tomography angiogram of chest showed a solid right axillary mass continuous with anterior margin of the right latissimus dorsi muscle (A, B and C); It measures $11 \mathrm{~cm} \mathrm{AP} \times 7.8 \mathrm{~cm}$ transverse $\times 10 \mathrm{~cm}$ craniocaudad diameter

\subsection{Operative report}

A Fine Needle Aspiration (FNA) biopsy of the right axillary/chest wall mass failed to reveal a definitive diagnosis. Operative excision of right axillary/chest wall mass with wide margins was carried out sparing right thoracodorsal nerve. The tumor had extensive vascularity in pericapsular region. The operation was complicated by the previous operative intervention in the right axilla for hyperhidrosis 10-15 years prior. Ultrasound was utilized to visualize the axillary vessels prior to incision. A radical excision was performed. The right axillary vein appeared to be invaded by tumor, requiring localized excision and ligation. The latisimus dorsi and teres major were also involved and were partially resected. An intraoperative frozen section was obtained and reported as a spindle cell lesion. There was extensive vascular supply to the mass. Following resection of this lesion, a Jackson-Pratt drain was left in the axilla. The patient was discharged to home the next day.

At two months follow-up, a PET scan revealed a $12 \mathrm{~mm}$ nodule in the left lower lobe with a SUV of 6.2 (see Figure 3). This nodule was not visualized in original CT scan of chest top months prior. The patient sub-sequently underwent a left video assisted thoracoscopic surgery and left lateral 
thoracotomy, in which an indurated nodule was found in the posterior basilar segment of the left lower lobe of the lung. A wide wedge resection of this nodule was performed with wide surgical margins. An intraoperative fro-zen section was obtained and reported as consistent with metastatic sarcoma.
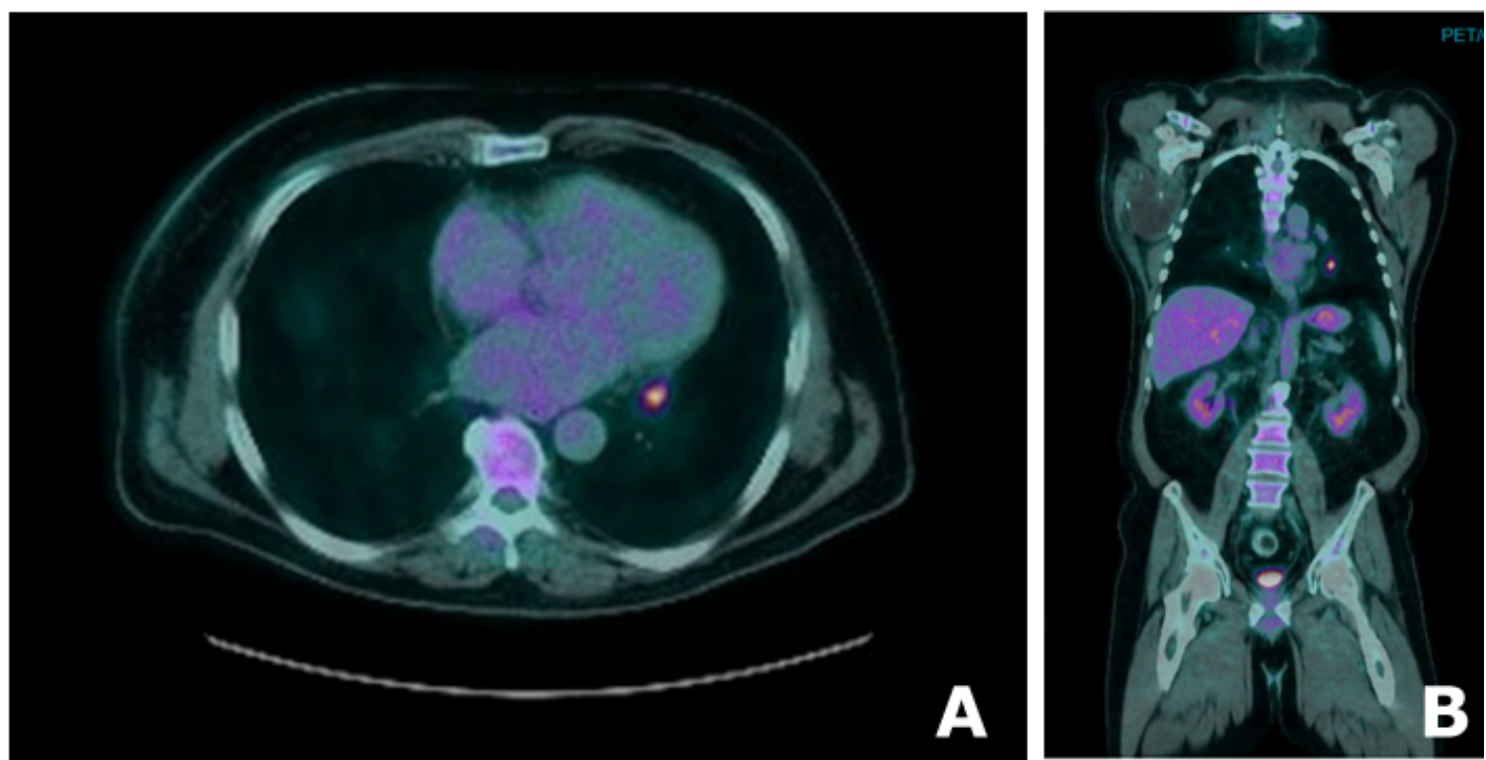

Figure 3. PET: $12 \mathrm{~mm}$ lobulated lung nodule is noted in the left lower lobe abutting the posterior cardiac border (A and B); This shows marked hypermetabolism measuring a maximum SUV of 6.2 suspicious for malignancy
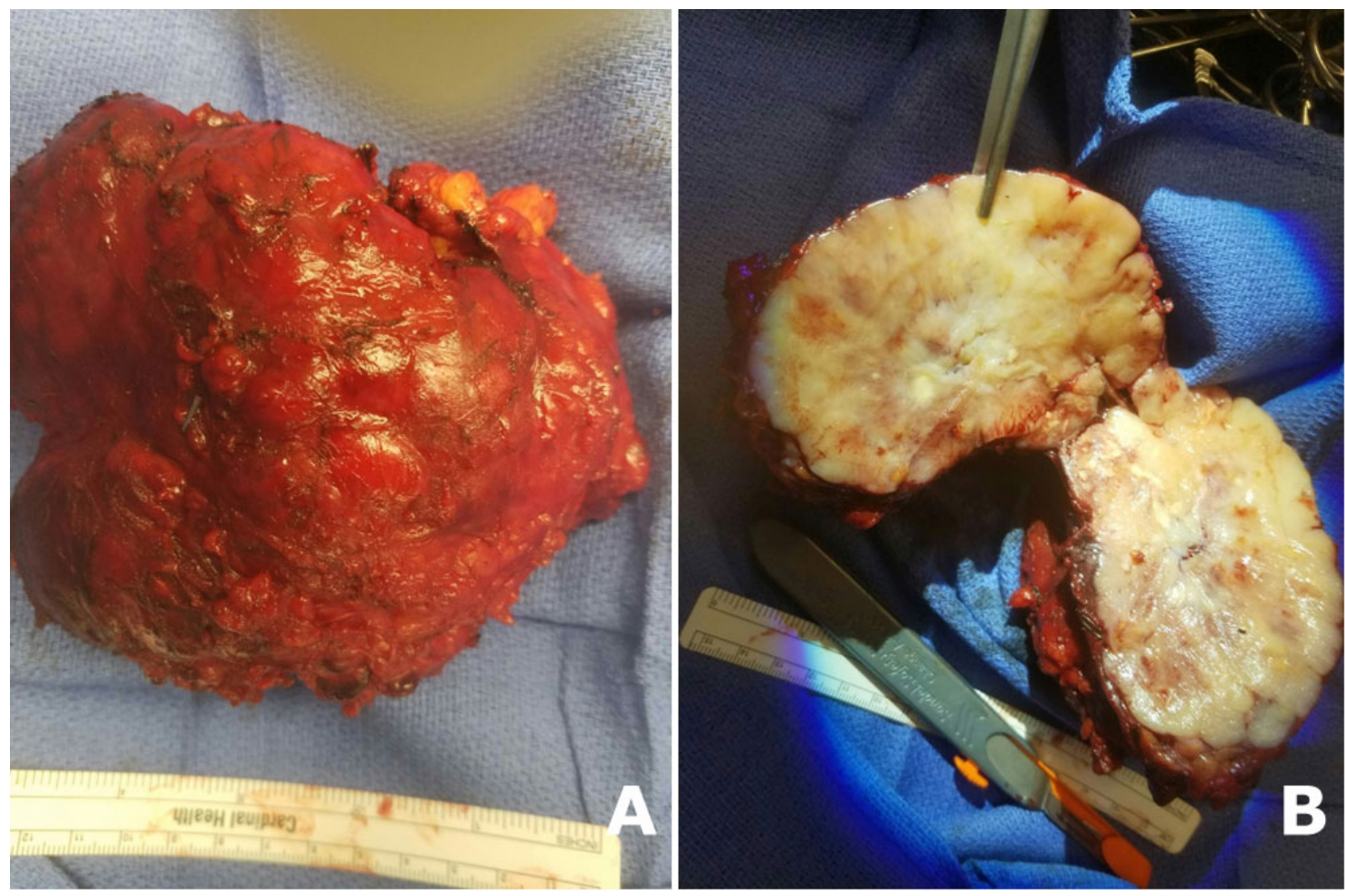

Figure 4. Gross section of resected tumor measuring $12 \mathrm{~cm} \times 10 \mathrm{~cm}$; Unsectioned mass (A) and cut surface of mass after sectioning $(\mathrm{B})$ 


\subsection{Pathology findings}

Histopathologic examination revealed a tumor measuring $12.8 \mathrm{~cm} \times 10.5 \mathrm{~cm} \times 7.6 \mathrm{~cm}$. Hematoxylin and Eosinstained sections demonstrated highly pleomorphic spindle cell tumor (see Figures 4 and 5). Foci of necrosis were also appreciated. Numerous atypical mitotic figures were noted throughout the tumor. Immuno-histochemical stains were performed showing that the tumor cells stained positively with S-100, p53, and vimentin. The tumor cells were negative for HMB-45, Melan-A, Keratin CAM 5.2, CD117 (c-KIT), CD34, desmin, smooth muscle cell actin, myogenin, calretinin, WT-1, CK5/6, CD57, synaptophysin, chromogranin, and Epithelial Membrane Antigen (EMA). These histomorphologic and immunophenotypic features are consistent with a high-grade pleomorphic liposarcoma.

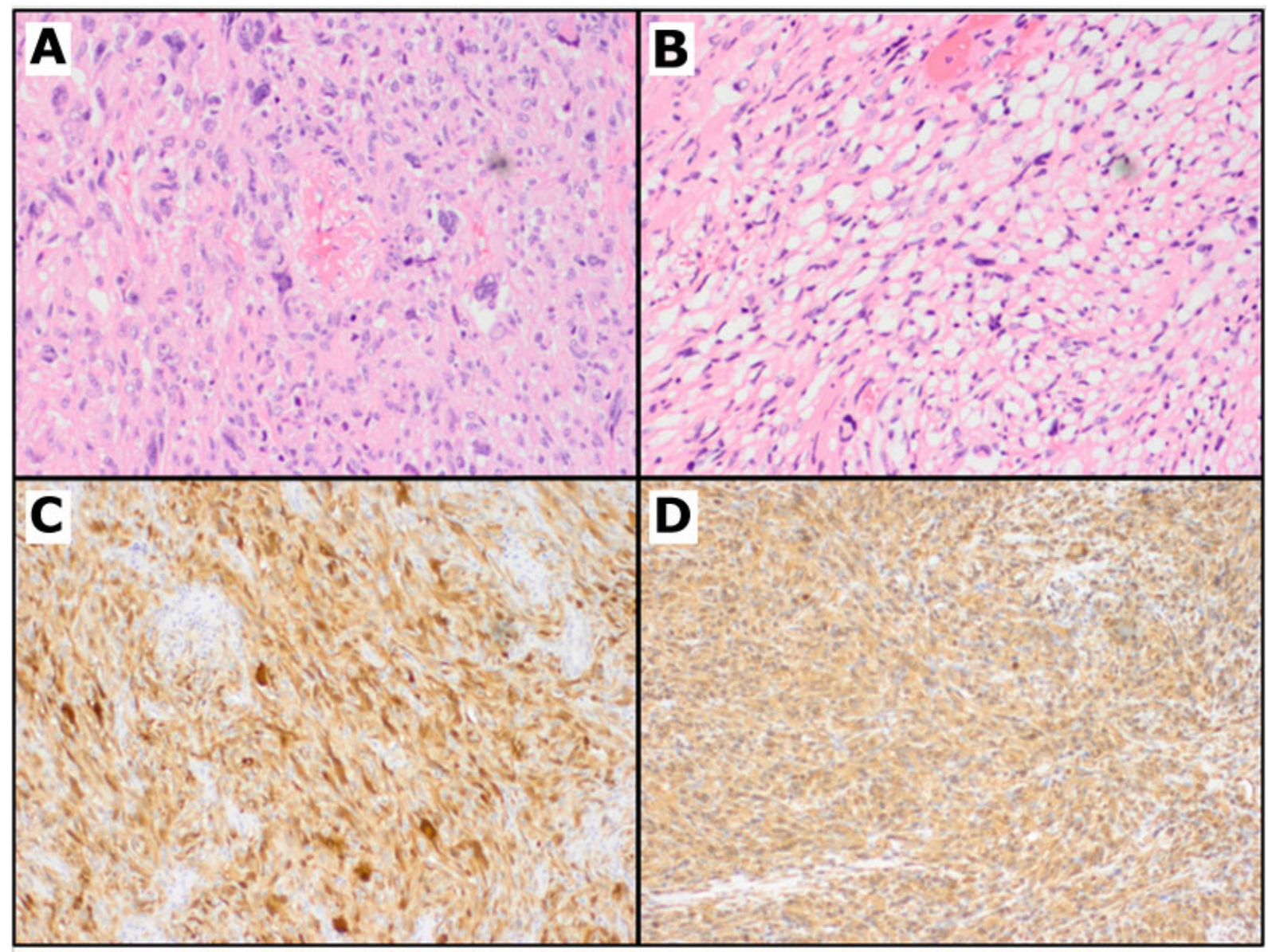

Figure 5. H\&E sections show sheets of large pleomorphic cells $(20 \times)(\mathrm{A})$; and other areas with more atypical lipomatous morphology (B); Immunohistochemical stains show these cells are positive for S100 (C) and Vimentin (D)

\section{Discussion}

Liposarcoma is a common malignant soft tissue tumor and accounts for approximately $20 \%-30 \%$ of soft tissue tumors. ${ }^{[1]}$ These lesions are most commonly encountered in adults in the $6^{\text {th }}$ or $7^{\text {th }}$ decade with cases very rarely reported in children. Liposarcoma is most frequently seen in retroperitoneal (45\%), extremity (24\%), inguinal, gluteal and popliteal regions. ${ }^{[2]}$ Chest wall or intrathoracic region tumors are extremely rare with few cases reported.

Clinicopathologic and cytogenetic sub-classification of liposarcoma includes well-differentiated, myxoid, pleomor- phic, and dedifferentiated types according to the $2012 \mathrm{NCCN}$ classification of liposarcoma. ${ }^{[3]}$ The finding of pleomorphic liposarcoma in the chest wall region is the rarest subtype of liposarcoma and is discriminated from other high-grade sarcomas by the presence of pleomorphic lipoblasts. ${ }^{[3]}$ It is a distinct and uncommon form and is often difficult to differentiate from other high-grade sarcomas. The current literature is limited in detailing its clinical and pathologic spectrum. From all reported studies of liposarcomas, only $4.5 \%$ were of pleomorphic type. ${ }^{[4]}$

As defined by the World Health Organization, pleomorphic liposarcomas show pleomorphic spindled and rounded 
cells with a variable number of pleomorphic lipoblasts on histopathology. ${ }^{[5]}$ Tumor necrosis is observed in $81 \%$ cases. Vascular invasion is rare, and mitotic counts range from 3-124 per 10 high power fields. Immunohistochemistry typically shows positive staining with Vimentin. S-100 positively can also be observed most commonly in lipogenic areas. Staining with CD34, CD68, smooth muscle actin, desmin, and Epithelial Membrane Antigen (EMA) can also be seen to varying degrees. ${ }^{[6,7]}$

In general, the differential diagnosis of pleomorphic liposarcoma includes pleomorphic leiomyosarcoma, pleomorphic rhabdomyosarcoma, pleomorphic malignant peripheral nerve sheath tumor, dedifferentiated angiosarcoma, and pleomorphic lipoma (benign). In cases such as this of a large axillary/chest wall mass, the differential diagnosis would also include metastatic carcinoma (for example, of lung or breast origin), metastatic melanoma, and lymphoma (Hodgkins and Non-Hodgkins). A panel of immunohistochemical markers and the recognition of pleomorphic lipoblasts may aid in confirming the diagnosis of pleomorphic liposarcoma. ${ }^{[8,9]}$

The paucity of cases of chest wall pleomorphic liposarcoma in the medical literature raises questions regarding appropriate treatment. Some studies report complete excision of well-differentiated liposarcomas and atypical lipomatous tumors due to high risk of local recurrence. ${ }^{[10]}$ Higher-grade liposarcoma in the retroperitoneum, where wide surgical excision is difficult to achieve, may receive adjuvant radiation therapy. All subtypes of liposarcoma will benefit from complete resection to minimize recurrence. ${ }^{[11,12]}$

Our case describes a large axillary/chest wall mass increasing in size significantly within a five e to six month period, eventually causing pain. A $12 \mathrm{~cm}$ mass was reported on MRI, and CT angiogram of the chest wall aided in defining axillary vein involvement. The patient subsequently underwent wide margin surgical resection of the mass, which was sent for histopathologic analysis which confirmed the diagnosis of pleomorphic liposarcoma. As described in our case, tumor location, size, immunohistochemical patterns, histopathologic appearance, mitotic rate, and the extent of necrosis together support the diagnosis of pleomorphic liposarcoma. The paucity of similar cases in the medical literature leaves many unanswered questions regarding potential treatment options. The possible role of neoadjuvant and adjuvant treatment, utilized in other types of high-grade liposarcomas, is still unknown for pleomorphic liposarcoma. Potential beneficial effects of adjuvant therapy are still unknown for this entity. One study reported recurrence of the tumor five months following wide surgical margin resection, and radiotherapy was subsequently added to prevent a new, local recurrence after reoperation. ${ }^{[13]}$ In our case, a frozen section of left lower lobe nodule from reoperation two months post-initial surgery was found to represent a metastatic pleomorphic liposarcoma. Adjuvant treatment with radiotherapy or chemotherapy may possibly be beneficial in this case to prevent further recurrence. There appears to be no data available regarding the effect of these treatment options on prognosis. Our future goal is to monitor this pleomorphic liposarcoma recurrence with close follow up and evaluate the effectiveness of combination therapy with wide margin surgical excision and adjuvant therapy. Due to the rarity of this diagnosis, any investigation into the most effective therapies for these patients would likely require the participation of multiple tertiary care centers.

\section{CONFLiCts OF InTEREST Disclosure}

The authors declare they have no conflicts of interest.

\section{REFERENCES}

[1] Cerullo G, Marrelli D, Rampone B, et al. Giant liposarcoma of the mesentery. Report of a case. Ann. Ital. Chir. 2007 Sep-Oct; 78(5): 443-445. PMid: 18338555.

[2] Weiss SW, Rao VK. Well-differentiated liposarcoma (atypical lipomatous tumors) of deep soft tissue of the extremities, retroperitoneum, and miscellaneous sites: a follow-up study of 92 cases with analysis of the incidence of dedifferentiation. Am J Surg Pathol. 1992; 16: 1051-1058. https://doi.org/10.1097/00000478-199211000 $-00003$

[3] Enzinger FM, Weiss SW. Liposarcoma. In: Soft tissue tumors. 3rd ed. St Louis, MO: Mosby; 1995. 431-466 p.

[4] Evans HL. Liposarcomas and atypical lipomatous tumours: a study of 66 cases followed for a minimum of 10 years. Surg Pathol. 1988; 1: $41-54$.

Published by Sciedu Press
[5] Fletcher CDM, Unni KK, Mertend F, editors. World Health Organization Classification of Tu-mours, Pathology and Genetics of Tumours of Soft Tissue and Bone. Lyon: IARC Press; 2002.

[6] Gebhard S, Coindre JM, Michels JJ, et al. Pleomorphic liposarcoma: clinicopathologic, immuno-histochemical, and follow-up analysis of 63 cases: a study from the French Federation of Cancer Centers Sarcoma Group. Am. J. Surg. Pathol. 2002 May; 26(5): 601-16. PMid: 11979090. https://doi.org/10.1097/00000478-200 205000-00006

[7] Suster S, Fisher C. Immunoreactivity for the human hematopoietic progenitor cell antigen (CD34) in lipomatous tumors. Am J Surg Pathol. 1998; 21: 195-200. https://doi.org/10.1097/000004 78-199702000-00009

[8] Suster S, Fisher C. Immunoreactivity for the human hematopoietic progenitor cell antigen (CD34) in lipomatous tumors. Am J Surg 
Pathol. 1998; 21: 195-200. PMid: 11266523. https://doi.org/ 10.1038/modpathol. 3880280

[9] Fletcher CDM. Pleomorphic malignant fibrous histiocytoma: fact or fiction? A critical reappraisal based on 159 tumors diagnosed as pleomorphic sarcoma. Am J Surg Pathol. 1992; 16: 213-228. PMid: 1317996. https://doi.org/10.1097/00000478-19920 3000-00001

[10] Mavrogenis AF, Lesensky J, Romagnoli C, et al. Atypical lipomatous tumors/well-differentiated liposarcomas: clinical outcome of 67 patients. Orthopedics. 2011; 34(12): e893-8. https://doi.org/ 10.3928/01477447-20111021-11
[11] Kilkenny JW, Bland KI, Copeland EM. Retroperitoneal sarcoma: the University of Florida experi-ence. J Am Coll Surg. 1996; 182(4): 329-39. PMid: 8605556.

[12] Matthyssens LE, Creytens D, Ceelen WP. Retroperitoneal liposarcoma: current insights in diag-nosis and treatment. Front Surg. 2015; 2: 4. PMid: 25713799. https://doi.org/10.3389/fsurg. 20 15.00004

[13] Fernandez EL, Plasencia LD, Palma JP, et al. Giant ulcerated pleomorphic liposarcoma of the chest wall. J. Thorac Oncol. 2007 Dec; 2(12): 1126-7. PMid: 18090587. https://doi.org/10.1097/JT $0.0 \mathrm{~b} 013 \mathrm{e} 31815 \mathrm{ba} 28 \mathrm{a}$ 